\title{
Liver Involvement Associated with Dengue Infection During A Major Outbreak in Central Nepal
}

\author{
Arun Sedhain, ${ }^{\text {a* }}$ Gandhi R Bhattarai, ${ }^{\mathrm{b}}$ Shital Adhikari, ${ }^{a}$ Barun Shrestha, ${ }^{\mathrm{a}}$ Abja Sapkota ${ }^{\mathrm{c}}$
}

${ }^{a}$ Department of Medicine, ${ }^{c}$ Lecturer, School of Nursing, Chitwan Medical College, Bharatpur, Chitwan Nepal, ${ }^{b}$ OptumHealth Care Solutions 400 Capital Boulevard, Rocky Hill, CT 06067, USA

\section{Accepted on \\ Jun $14^{\text {th }}, 2013$}

\section{DOI Name}

http://dx.doi.org/10.3126/jaim.v2i2.8775

\section{Keywords}

Dengue fever, dengue hemorrhagic fever, hepatic manifestations

\section{Citation}

Arun Sedhain, Gandhi R Bhattarai, Shital Adhikari, Barun Shrestha, Abja Sapkota. Liver Involvement Associated with Dengue Infection During A Major Outbreak in Central Nepal. Journal of Advances in Internal Medicine 2013;02(02):42-6.

\begin{abstract}
Background and Aims - Dengue is a systemic viral infection, which is caused by any of the four strains of dengue virus and is transmitted between humans by the bite of Aides aegypti mosquito. Hepatic dysfunction is common in dengue infection with varying degree according to the severity of the disease. The aim of this study was to analyze the spectrum of liver involvement in dengue infection during a major outbreak that occurred in central Nepal between September and December 2010.
\end{abstract}

Methods - A hospital based prospective study was conducted in the department of medicine at Chitwan Medical College. Patients who had fever in the ten preceding days (acute febrile illness) who had serologic evidence of Dengue and had no clinical evidence of bacterial infection anywhere in the body, were included in the study. Clinical, biochemical and radiological evaluation was done among 414 serologically confirmed Dengue cases to assess the spectrum of liver involvement. Statistical analysis of the data was done with SPSS version 16.

Results - Dengue fever (DF) and dengue hemorrhagic fever (DHF) were present in $79.3 \%$ and $20.5 \%$ of the patients respectively. Only 2 out of 414 patients had dengue shock syndrome (DSS). The spectrum of hepatic manifestations included elevation of transaminases (raised ALT and AST in $81.9 \%$ and $57.7 \%$ respectively), abnormal abdominal ultrasound (49.3\%), Hepatomegaly (39.1\%), and Jaundice (4.1\%). The intensity of hepatic involvement was significantly higher among patients with DHF than those with DF.

Conclusion: Liver is one of the major organs to be involved in Dengue infection. Significant difference existed in the spectrum of liver involvement between DF and DHF.

\section{INTRODUCTION}

Dengue is one of the most important arboviral infections of humans. ${ }^{1}$ The disease is caused by four antigenically distinct but related dengue viruses (DENV 1-4) and is transmitted primarily by Aedes aegypti mosquito. The global incidence of DF and DHF has increased dramatically in recent decades. ${ }^{1,2}$ Dengue virus is now the most common cause of arboviral disease in the world, with an estimated annual occurrence of 100 million cases of DF and 250000 cases of DHF with a mortality rate of 25000 per year. ${ }^{3}$

The spectrum of Dengue infection has been conventionally divided into Dengue Fever (DF), Dengue Hemorrhagic Fever (DHF) and Dengue Shock Syndrome (DSS). Unusual manifestations involving liver and central nervous system in

\footnotetext{
* Corresponding author

Dr. Arun Sedhain

Department of Nephrology

National Academy of Medical Sciences (NAMS), Bir Hospital

Kathmandu, Nepal

Email: purkang@gmail.com
} 
dengue infection have been reported. ${ }^{4,5}$ Hepatic dysfunction is a well-recognized feature of dengue infections, often demonstrated by hepatomegaly and mild-to moderate increases in transaminase levels although jaundice and acute liver failure are generally less common. ${ }^{6,7}$ However, there are few case reports of fulminant hepatic failure in DHF. ${ }^{8,9}$ The incidence of hepatic dysfunction is more in DHF than in DF. ${ }^{4}$ This study aims at analyzing the spectrum of liver involvement between DF and DHF using the results obtained during a major dengue outbreak in Nepal.

\section{METHODS}

A major dengue outbreak occurred in Chitwan district and surrounding areas between September and December, 2010. A prospective study was conducted to describe the spectrum of symptomatic dengue disease among adults admitted to the department of medicine at Chitwan Medical College. During this time, patients with a history of fever in the ten preceding days (acute febrile illness) and no clinical evidence of bacterial infection anywhere in the body were examined and had at least one blood sample collected for specific dengue and other laboratory tests. Among the 1456 patients tested for dengue serology, 426 tested positive for the disease of which 414 cases were included in the study as rest of the patients $(n=12)$ had other concomitant conditions like viral hepatitis, malaria and leptospirosis.

The definition of DHF included fever, thrombocytopenia (platelet count less than 100000 per $\mathrm{mm}^{3}$ ), hemorrhagic manifestations, and objective evidence of plasma leakage revealed by more than $20 \%$ rise in hematocrit over baseline or more than $20 \%$ drop in hematocrit after treatment or presence of plasma leakage signs such as pleural effusion, ascites and hypoproteinemia. ${ }^{1}$ For the diagnosis of dengue shock syndrome (DSS), the presence of hypotension or narrow pulse pressure was required in addition to the previously mentioned criteria. Any subject with serological evidence of acute dengue infection who did not meet the criteria for DHF or DSS was considered as having DF.

After written informed consent, demographic and personal details of the patients were recorded. Clinical data were collected through interviews with patients or their attendants. Detailed clinical information were recorded with special focus on the duration and intensity of fever, as well as associated symptoms like headache, nausea, vomiting, anorexia, body ache and bleeding diathesis. A meticulous physical examination of the patients was conducted. Hepatomegaly and ascites were ascertained by physical examination and on reports of ultrasonography and $\mathrm{X}$-rays.
All blood samples were tested for dengue virus IgM or IgG antibodies. SD Dengue IgM/IgG capture ELISA kit and SD Dengue IgM/IgG rapid test kit, both produced by Standard Diagnostics Inc., Kyonggi-do, Korea, was used for testing in the laboratory. In the initial days of the study, when there was a limitation of resources and testing mechanism, rapid test for dengue was applied. Subsequently, rapid IgM-IgG capture ELISA test, which has become the standard for serological diagnosis of dengue fever, ${ }^{10}$ was initiated. Most of the laboratory tests were done at the National Reference Laboratory (NRL) located within the Chitwan Medical College Hospital. Additional laboratory investigations carried out in these patients included hemoglobin, total and differential leucocyte count, platelet count and liver function tests. Chest $X$-ray and ultrasound of the abdomen were done whenever clinically indicated. Statistical analysis of the data was done with SPSS version 16.

\section{RESULTS}

More than two third of the patients came from Chitwan district (70.4\%) followed by Nawalparasi (18.8\%) and Rupandehi districts (6.0\%). Table 1 shows the district-wise distribution of dengue patients by their place of residence.

Table 1: District-wise distribution of dengue patients

\begin{tabular}{lll}
\hline District & No. of cases & Percentage \\
\hline Chitwan & 291 & $70.36 \%$ \\
Nawalparasi & 78 & $18.79 \%$ \\
Rupandehi & 25 & $6.02 \%$ \\
Tanahun & 11 & $2.65 \%$ \\
Makawanpur & 6 & $1.44 \%$ \\
Parsa & 2 & $0.48 \%$ \\
Lamjung & 1 & $0.24 \%$ \\
Lamjung & 1 & $0.24 \%$ \\
\hline
\end{tabular}

A majority of the patients (84.6\%) were in the age group 16-60 years, which is considered to be the most productive part of one's life (Figure 1).Total number of male and female patients was 242 and 172 respectively with the Male: Female ratio of 1: 0.7 .

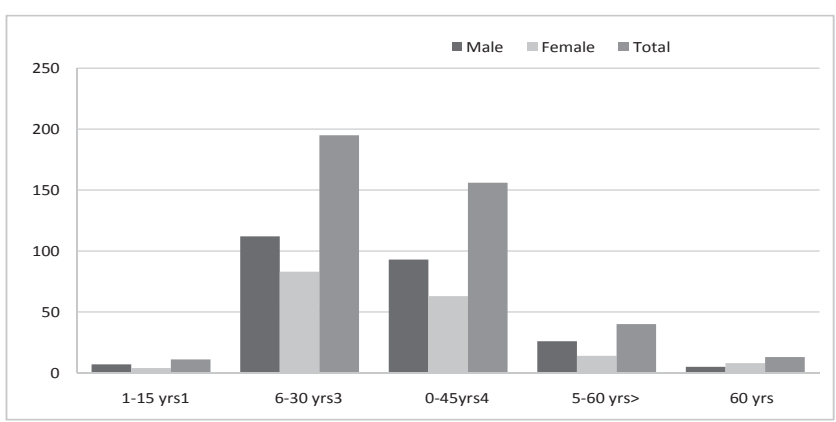

Figure 1: Age and sex wise distribution of patients 
Table 2 indicates the signs and symptoms of all patients who presented with fever and were diagnosed with DF. Major common symptoms included headache (96.6\%), body ache (93.1\%), nausea (84.6\%) and vomiting (62.8\%). Other important symptoms included retro-orbital pain (49.2\%), itching $(42.7 \%)$, abdominal pain (41.9\%) and hepatomegaly (39.1\%). Complete list of symptoms are presented in Table 2.

Table 2: Symptomatic presentations of dengue patients

\begin{tabular}{lc}
\hline Clinical manifestation & Number of patients (\%) \\
\hline Fever & $414(100 \%)$ \\
Headache & $401(96.62 \%)$ \\
Body ache & $386(93.06 \%)$ \\
Nausea & $351(84.57 \%)$ \\
Vomiting & $260(62.79 \%)$ \\
Retro-orbital pain & $204(49.15 \%)$ \\
Itching & $177(42.65 \%)$ \\
Abdominal pain & $174(41.93 \%)$ \\
Hepatomegaly & $162(39.06 \%)$ \\
Skin rash & $111(26.74 \%)$ \\
Loose motion & $103(24.81 \%)$ \\
Splenomegaly & $99(23.85 \%)$ \\
\hline Jaundice & $67(16.14 \%)$ \\
\hline
\end{tabular}

Following the existing WHO criteria, $79.3 \%(n=329)$ of the patients were diagnosed with classic DF and $20.5 \%(n=85)$ with DHF. Three of them had clinically significant bleeding. One of the two patients diagnosed with DSS had co-morbidities of post-TB bronchiectasis and alcoholic liver disease and died during the course of the treatment in the ICU. One of the patients was admitted with features of fulminant hepatic failure with altered sensorium, deep jaundice and deranged transaminase level (AST of $14100 \mathrm{IU}$ and ALT of $5760 \mathrm{IU}$ ). However, this patient survived and AST and ALT values returned to $105 \mathrm{IU}$ and $120 \mathrm{IU}$ respectively on the eleventh day of illness. All three measures of platelet counts, elevated ALT and elevated AST were significantly higher in DHF as compared to patients with DF.
Table 3: Laboratory parameters of DF and DHF patients.

\begin{tabular}{lcccccc}
\hline \multirow{2}{*}{ Variables } & \multicolumn{2}{c}{ DF $(\mathbf{n = 3 2 9})$} & \multicolumn{2}{c}{ DHF $(\mathbf{n}=85)$} & P value \\
& Count & Percentage & Count & Percentage & \\
\hline $\begin{array}{l}\text { Platelet } \\
<50000\end{array}$ & 32 & $9.73 \%$ & 43 & $51.19 \%$ & $<.0001$ \\
$\begin{array}{l}\text { ALT more } \\
\text { than 50 }\end{array}$ & 258 & $78.42 \%$ & 81 & $96.43 \%$ & $<.0001$ \\
$\begin{array}{l}\text { AST more } \\
\text { than 50 }\end{array}$ & 177 & $53.80 \%$ & 62 & $73.81 \%$ & $<.0001$ \\
\hline
\end{tabular}

The results from ultrasound of the abdomen including hepatomagaly, thickened gall bladder and third space loss are presented in Table 4. All three measures were significantly higher in DHF as compared to patients with DF. Hepatomegaly was present in $72.9 \%$ DHF cases as compared to only $30.4 \%$ cases with DF. Similarly, thickening of gall bladder was higher in DHF (25.9\%) as compared DF (6.1\%). Third space loss was present all but one case of DHF (98.8\%) where as it was present only in $5.5 \%$ of DF cases.

Table 4: Ultrasonologic manifestations of DF and DHF patients.

\begin{tabular}{|c|c|c|c|c|c|}
\hline \multirow{2}{*}{ Variables } & \multicolumn{2}{|c|}{$D F(n=329)$} & \multicolumn{2}{|c|}{ DHF $(n=85)$} & \multirow{2}{*}{$\begin{array}{c}P \\
\text { value }\end{array}$} \\
\hline & Count & Percentage & Count & Percentage & \\
\hline Hepatomegaly & 100 & $30.40 \%$ & 62 & $72.94 \%$ & $<.0001$ \\
\hline $\begin{array}{l}\text { Thickened gall } \\
\text { bladder }\end{array}$ & 20 & $6.08 \%$ & 22 & $25.88 \%$ & $<.0001$ \\
\hline $\begin{array}{l}\text { Third space } \\
\text { loss }\end{array}$ & 18 & $5.47 \%$ & 84 & $98.82 \%$ & $<.0001$ \\
\hline
\end{tabular}

\section{DISCUSSION}

The dengue outbreak started between end-August and early September and peaked by late October. Dengue-specific IgM was positive in $69 \%$ of patients, which is comparable to other studies. ${ }^{11,12}$ According to WHO criteria for diagnosis, $329(79.3 \%)$ of the patients were diagnosed as classic DF, 83 (20.5\%) as DHF, with additional two DSS cases.

Hepatic involvement in dengue infections is often demonstrated by hepatomegaly and mild-to- moderate increases in transaminase levels. Clinical evidence included hepatomegaly and increased serum liver enzymes, with liver involvement being more pronounced in the more severe forms of infection. Out of 414 cases, $39.1 \%$ had hepatomegaly. The difference in hepatomegaly among DF and DHF was significant $(p<.0001)$ which was more common in DHF $(72.9 \%)$ than in DF (30.4\%). In one study done in India, $79 \%$ of the patients had hepatomegaly. ${ }^{17}$ Another study done in Vietnam revealed 
hepatomegaly in $96 \%$ of Dengue patients and also showed significant differences in hepatic changes among those with and without shock. ${ }^{18}$

In dengue infections, elevations in serum AST appear to be greater than ALT levels. This differs from the pattern in viral hepatitis, in which ALT levels are usually higher than or equal to AST levels. ${ }^{13}$ The causes from these indications are still uncertain. It has been suggested that it may be due to excess release of AST from damaged myocytes during dengue infections. ${ }^{14}$ The elevated AST levels tend to return to normal more rapidly than ALT levels. This is possibly because AST $\left(12.5-22 \mathrm{~h}\right.$ ) has a shorter half-life than ALT (32-43 h). ${ }^{15}$

In the study, liver enzyme AST was increased with the value more than $50 \mathrm{IU} / \mathrm{ml}$ in $78.4 \%$ and $96.5 \%$ of cases of DF and DHF respectively. Similarly, ALT value was raised in $53.8 \%$ of DF and $74.1 \%$ of DHF patients. The difference in the rise of liver enzymes in DHF and DF was statistically significant $(P<0.001)$. Various studies in the past reported liver dysfunction to be more common in DHF than in DF patients. ${ }^{17-19}$

The dengue virus is able to replicate in both hepatocytes and Kupffer cells. ${ }^{16}$ Mechanisms of liver injury in dengue may be due to direct effects of the virus or host immune response on liver cells, circulatory compromise, metabolic acidosis and/or hypoxia caused by hypotension or localized vascular leakage inside the liver. ${ }^{22-24}$

Detection of abnormally high transaminase enzymes among patients with dengue is important since the possibility of consequent hepatic encephalopathy can be expected. ${ }^{4}$ Liver dysfunction was mild to moderate in most of the patients in the study, presenting primarily as elevation of serum aminotransferases. However, some patients had clinical manifestations of liver disease, namely jaundice, hepatomegaly and ascites. One of the patients with DSS had findings consistent with acute liver failure with AST and ALT level of 14100 IU and 5760 IU respectively. However, the patient survived and had near normal aminotransferase level at the time of discharge, which had come down to normal in follow up after one month.

The most common ultrasound findings were hepatomegaly, third space loss in the form of ascites and pleural effusion and thickened gall-bladder. DHF had significantly higher prevalence of these conditions then DF. Similar findings in ultrasound abdomen were reported in different studies. ${ }^{20,21}$

\section{CONCLUSION}

Dengue, which was considered a rare disease entity in Nepalese context, is not a strange disease. The spectrum of liver involvement in dengue varied as per the severity of dengue infection. The study findings suggest that presence of fever, jaundice and hepatomegaly in endemic areas should arouse the suspicion of hepatitis associated with dengue infection.

hepatic failure in an infant with severe Dengue infection. Indian J Pediatr 2010;77:435-7.

6. Souza LJ , Alves JG , Nogueira RM , et al. Aminotransferase changes and acute hepatitis in patients with dengue fever: analysis of 1,585 cases. Braz J Infect Dis 2004;8:156 63 .

7. Mohan B , Patwari AK, Anand VK. Hepatic dysfunction in childhood dengue infection. J Trop Pediatr 2000;46:40-3.

8. Sedhain A, Adhikari S, Regmi S, et al. Fulminant Hepatic Failure due to Dengue. Kathmandu Univ Med J 2011;34:73-5.

9. Lawn SD, Tilley R, Lloyd G, et al. Dengue hemorrhagic fever with fulminant hepatic failure in an immigrant returning to Bangladesh. Clin Infect Dis 2003; 37:e1-e4.

10. Narayanan $\mathrm{M}$, Aravind MA, Thilothammal $\mathrm{N}$, et al Dengue fever epidemic in Chennai - a study of clinical profile and outcome. Indian Pediatr 2002;39:1027-33.

5. Soundravally R, Narayanan P, BhatVB, et al. Fulminant 
11. Gupta E, Dar L, Narang P, et al. Serodiagnosis of dengue during an outbreak at a tertiary care hospital in Delhi. Indian J Med Res 2005;121:36-8.

12. Kishore J, Singh J, Dhole TN, et al. Clinical and serological study of first large epidemic of dengue in and around Lucknow, India, in 2003. Dengue Bulletin 2006;43:72-9.

13. Gholson CF, Provenza JM, Bacon BR, et al. Hepatologic considerations in patients with parenchymal liver disease undergoing surgery. Am J Gastroenterol 1990;85:487-96.

14. Chung HK, Dar IT, Chi SC, et al. Liver biochemical tests and dengue fever. Am J Trop Med Hyg 1992;47:265-70.

15. Hawker F. Liver dysfunction in critical illness. Anaesth Intensive Care 1991;19: 165-81.

16. Huerre MR, Lan NT, Marianneau P, et al. Liver histopathology and biological correlates in five cases of fatal dengue fever in Vietnamese children. Virchows Arch 2001;438:107-15.

17. Jagadishkumar K, Jain P, Manjunath VG, et al. hepatic failure in dengue fever in children. Iran J Pediatr 2012;22:231-6.

18. Trung DT, Thao LTT, Hien TT, et al. Liver involvement associated with dengue infection in adults in Vietnam Am J Trop Med Hyg 2010;83:774-80.

19. Wahid SF, Sansui S, Zawawi MM, et al. A comparison of the pattern of liver involvement in dengue hemorrhagic fever with classical dengue fever. Southeast Asian J Trop Med Public Health 2000;31:259-63.

20. Sharma S, Sharma SK, Mohan A, et al. Clinical profile of Dengue Haemorrhagic fever in adults during 1996-outbreak in Delhi, India. Dengue Bulletin 1998:22;20-30.

21. Aikat BK, Konar NR, Banerjee G. Hemorrhagic fever in Calcutta area. Indian J Med Res 1964;52:660-75.

22. Mohan B, Patwari AK, Anand VK. Hepatic dysfunction in childhood dengue infections. J Trop Pediatr 2000;46:403.

23. Itha S, Kashyap R, Krishnani N, et al. Profile of liver involvement in dengue virus infection. Natl Med J India 2005;18:127-30.

24. Seneviratne SL, Malavige GN, de Silva HJ. Pathogenesis of liver involvement during dengue viral infections. Trans R Soc Trop Med Hyg2006;100:608-14. 